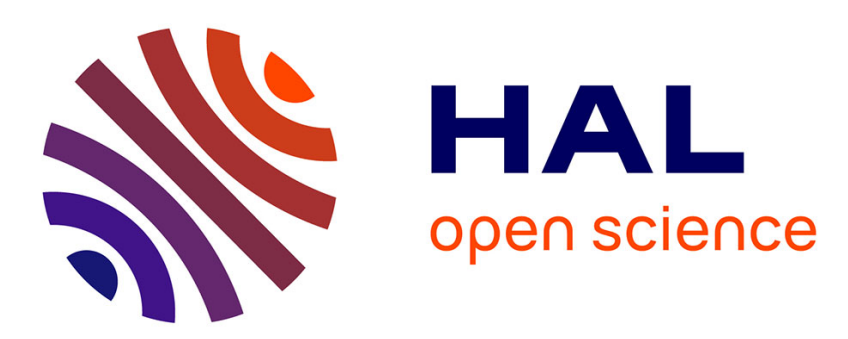

\title{
La forme juridique du futur accord de Paris sur le climat : enjeux et principales options
}

Sandrine Maljean-Dubois, Thomas Spencer Spencer, Matthieu Wemaere

\section{To cite this version:}

Sandrine Maljean-Dubois, Thomas Spencer Spencer, Matthieu Wemaere. La forme juridique du futur accord de Paris sur le climat: enjeux et principales options . Cahiers, Droit, Sciences \& Technologies, 2015. halshs-01311785

\section{HAL Id: halshs-01311785 \\ https://shs.hal.science/halshs-01311785}

Submitted on 4 May 2016

HAL is a multi-disciplinary open access archive for the deposit and dissemination of scientific research documents, whether they are published or not. The documents may come from teaching and research institutions in France or abroad, or from public or private research centers.
L'archive ouverte pluridisciplinaire HAL, est destinée au dépôt et à la diffusion de documents scientifiques de niveau recherche, publiés ou non, émanant des établissements d'enseignement et de recherche français ou étrangers, des laboratoires publics ou privés. 


\title{
La forme juridique du futur accord de Paris sur le climat : enjeux et principales options ${ }^{(*)}$
}

\author{
Sandrine Maljean-Dubois \\ Directrice de recherche CNRS \\ (CERIC UMR 7318 DICE, CNRS, Aix-Marseille Université)
}

\author{
Thomas Spencer \\ Directeur du programme énergie et climat, \\ Institut du développement durable et des relations internationales (Iddri), Paris \\ Matthieu Wemaëre \\ Avocat au barreau de Paris et Bruxelles, \\ Chercheur associé au CERIC \\ (UMR 7318 DICE, CNRS, Aix-Marseille Université)
}

\begin{abstract}
Résumé
Depuis plusieurs années, la question de la forme juridique du futur accord climatique mondial plane sur les négociations. Les Parties ont insisté pour discuter d'abord de la substance de l'accord. II est vrai que de larges divergences demeurent de ce point de vue. Cependant, à quelques mois de la conférence de Paris destinée à adopter cet accord, il est temps de réfléchir à la forme juridique qu'il prendra. Or, la question de la forme juridique est bien plus complexe qu'elle n'y paraît à première vue. D'une part, elle ne se résume pas au choix binaire entre un traité ou un instrument non obligatoire. D'autre part, elle soulève à son tour de nombreuses questions, dont les réponses détermineront la capacité du futur accord de Paris à limiter les changements climatiques et le réchauffement global de la planète de manière effective. Elles tiennent à la nature et portée des engagements ou promesses de réduction des émissions, au suivi des émissions, à un éventuel contrôle international des engagements ou promesses, voire à une sanction du nonrespect de ceux-ci. Ces différents paramètres sont liés et méritent d'être examinés ensemble, pour avoir une vision globale des différentes options juridiques et de leurs conséquences sur le choix de la forme juridique de l'accord de Paris.
\end{abstract}

\begin{abstract}
For many years, the issue of the legal form of the new climate agreement has hovered over the international negotiations. Countries have insisted on first discussing substance. Indeed, it is here that the main divergences remain. However, less than one year before the Paris climate conference, it is time to open the discussion on the legal form of the final agreement. The issue of the legal form is far more complex than at first sight. It is often reduced to the negotiation of a 'binding' or 'non-binding' agreement. The bindingness of an international environmental agreement however depends on multiple parameters. We propose four parameters to be considered: the form of the core agreement; the 'anchoring' of commitments; mechanisms for transparency, accountability and facilitation; and mechanisms for compliance. Parties should assess pros and cons of these options, and the agreement be optimised across all four.
\end{abstract}

\section{Introduction}

$\left(^{*}\right)$ Les auteurs remercient l'Iddri pour le soutien accordé à cette étude dont une version préliminaire a été publiée en anglais sous la forme d'un working paper de l'Iddri " A comprehensive assessment of options for the legal form of the Paris Climate Agreement », Working Papers Nº15/2014. Iddri, 2014. 20 p. 
La réduction des émissions de gaz à effet de serre constitue l'enjeu principal des négociations, lancées à Durban en 2011, qui doivent aboutir en décembre 2015 à l'adoption d'un accord à Paris, lors de la 21e Conférence des Parties (COP) à la Convention-cadre sur les changements climatiques (CCNUCC) de 1992, lequel accord sera le cœur du régime international sur le climat à partir de 2020. En effet, à cette date, la deuxième période d'engagement du Protocole de Kyoto aura pris fin sans que ne soit envisagée/négociée une troisième période. Les promesses de réduction des émissions faites en application de l'Accord de Copenhague, dans le cadre plus inclusif de la Convention, ne vont pas non plus au-delà de 2020. Les États ne sont donc pas, ni dans un cadre, ni dans l'autre, engagés à réduire leurs émissions de gaz à effet de serre au-delà.

À Durban, les Parties sont convenues d'« élaborer au titre de la Convention un protocole, un autre instrument juridique ou un texte convenu d'un commun accord ayant valeur juridique, applicable à toutes les Parties ${ }^{1}$. La question de la forme de l'accord de Paris n'a donc pas été tranchée. Elle ne l'a pas davantage été depuis lors. Même si une majorité de Parties semble s'accorder sur un ensemble constitué d'un accord juridiquement obligatoire - un Protocole relativement concis et bref, complété par un paquet de décisions des Parties, cette option est loin de faire l'unanimité.

Mais les négociations ont avancé en substance et un texte de négociation est même sur la table depuis la Conférence de Lima ${ }^{2}$. Dans le cadre d'un accord qui devra être "applicable à toutes les Parties " ${ }^{3}$, les Parties sont toutes "invitées " à préparer leurs " contributions prévues " à l'effort collectif, fruits de " préparatifs internes » et « déterminées au niveau national » (INDCs en anglais pour "intended nationally determined contributions »), selon un calendrier relativement serré puisqu'elles doivent « en faire part bien avant la vingt et unième session de la Conférence des Parties (d'ici au premier trimestre 2015 pour les Parties qui sont prêtes à le faire) d'une manière propre à améliorer la clarté, la transparence et la compréhension des contributions prévues ». À deux reprises, la décision prend soin de préciser que c'est "sans préjudice de la nature juridique desdites contributions ${ }^{4}$. Ainsi, alors qu'un consensus semble progresser vers l'adoption d'un nouveau protocole à la Convention de 1992, quelle sera la nature juridique des " contributions » des États ? Vont-elles faire l'objet d'une reconnaissance voire d'un engagement international ? Figurer dans le protocole lui-même et, si oui, dans son corps ou dans une annexe? Figureront-elles seulement dans une décision de la Conférence des Parties qui viendra, ou non, compléter le protocole? Ou encore dans un document sans aucune valeur juridique comme c'est le cas des "promesses » de Copenhague ? Que signifie par ailleurs "prévues »? Y aura-t-il place après Paris pour un processus d'évaluation internationale des contributions nationales, avec à la clé une possible incitation à un relèvement du niveau d'ambition ? Et, en ce cas, comment faire en sorte que la forme juridique des contributions ne contribue pas à figer un niveau d'ambition relativement bas ? Quid par ailleurs des revendications d'équité ou justice internationale en application du principe des responsabilités communes, mais différenciées et des capacités respectives, clairement inscrit dans la Convention-cadre de $1992^{5}$ ? II est acquis que le futur accord devra être "applicable à tous" comme l'exige la Plateforme de Durban. Mais quelle différenciation autorisera le futur accord de Paris ? La différenciation se traduira-t-elle simplement par des contributions nationales différenciées ? Ou ira-t-elle au-delà ?

De fait, la question de la forme juridique du futur accord est bien plus complexe que ce qu'il n'y paraît à première vue. D'une part, elle ne se résume pas au choix binaire entre un traité ou un instrument non obligatoire. D'autre part, elle soulève à son tour de nombreuses questions, dont les réponses détermineront la capacité du futur accord de Paris à limiter les changements climatiques et le réchauffement global de la planète. Elles tiennent à la nature et portée des engagements ou promesses de réduction des émissions, au suivi des émissions, à un éventuel contrôle international des réductions, voire à une sanction du non-respect. Ces différents paramètres sont liés et méritent d'être examinés ensemble, pour avoir une vision globale des différentes options juridiques et de leurs conséquences.

\footnotetext{
${ }^{1}$ Décision 1/CP.17, Création d'un groupe de travail spécial de la plate-forme de Durban pour une action renforcée (2011)

${ }^{2}$ Version revue à Genève en février 2015, https://unfccc.int/files/bodies/awg/application/pdf/negotiating text 12022015@2200.pdf consulté le 6 mars 2015

3 Ibid., §2.

${ }^{4}$ Décision 1/CP.19, Moyens de poursuivre la mise en œuvre de la plate-forme de Durban (2013).

${ }^{5}$ Article $3 \S 1$.
} 


\section{Remarques introductives}

\section{normativité}

1.1. Hard et soft-law en droit international de l'environnement: les critères de la

À l'opposé des visions binaires opposant le droit au non-droit sans entre-deux, force est de constater que la densité normative du droit international de l'environnement est extrêmement variable.

Le premier constat d'évidence est que la soft-law y est à la fois abondante et multiforme. D'abord, le qualificatif de soft ne concerne pas seulement la source formelle. II peut viser aussi bien une obligation conventionnelle relativement souple, qu'un instrument non obligatoire ou bien à l'« obligatoriété » discutée. Dans le champ de l'environnement, nombreuses sont par exemple les obligations conventionnelles atténuées au point d'en devenir fuyantes et insaisissables. Nombreuses sont les propositions normatives non obligatoires, car non rédigées comme des normes prescriptives ou bien en des termes si généraux que cela les rend non applicables en l'absence de précisions complémentaires.

Ceci dit, le qualificatif de soft peut englober un grand nombre d'instruments très différents à la fois par leur nature et leur contenu : résolutions d'organisations internationales, droit dérivé des conventions internationales de protection de l'environnement, déclarations (type Déclaration de Rio de 1992), normes techniques, standards, memoranda of understanding, stratégies et plans d'action, codes de conduite, partenariats publics-privés, etc. Au regard des sources formelles classiques (traités et coutumes), ces instruments présentent différents avantages. Ils permettent des gains en souplesse, rapidité et évolutivité. Certains peuvent être conclus par des acteurs qui ne se sont pas vu reconnaître la qualité de sujet du droit international (par exemple un memorandum of understanding entre deux secrétariats de conventions internationales; partenariats publics-privés).

Les instruments de soft-law sont a priori non obligatoires mais ils peuvent avoir une certaine valeur juridique en pratique : le soin mis à négocier le contenu de tels actes, tout comme le fait que les États acceptent parfois l'instauration de mécanismes de suivi et de contrôle de leur application $^{6}$, en fournissent des indices assez sûrs. Les degrés de normativité et d'effectivité des instruments de soft-law sont en réalité variables. La summa divisio entre le hard et le soft, entre l'obligatoire et le non-obligatoire, ne résiste en tous cas pas à une analyse approfondie ${ }^{7}$. Doit-on voir des symptômes pathologiques dans cette normativité variable ${ }^{8}$, comprenant d'un côté de l'échelle des normes à autorité renforcée (jus cogens et erga omnes) et de l'autre des normes de nature et qualité normative « assourdie $»^{9}$, que certains décrivent comme des twilight norms $\|^{10}$ ?

La soft-law peut se présenter comme du pré-droit ou "droit vert " in statu nascendi, un creuset pour le droit positif, qu'elle marque une étape dans la formation d'une règle conventionnelle, ou révèle l'existence d'une règle coutumière. II suffit ici de se référer au rôle considérable joué dans le développement ultérieur du droit international de l'environnement par les Déclarations de Stockholm en 1972 ou Rio en 1992, ou encore la Charte mondiale de la nature en $1982^{11}$. Ces grands textes du droit international de l'environnement ont préparé l'adoption de règles conventionnelles, et cristallisé ou contribué à la cristallisation, voire révélé l'existence, de règles coutumières nouvelles. On peut penser aussi à de nombreuses lignes directrices qui sont devenues dans plusieurs cas des conventions internationales. Comme le résume G. Abi-Saab, "La soft-law sert ainsi de curseur et de locomotive au processus dynamique et cumulatif du développement du droit et jalonne son passage à travers la zone grise ${ }^{12}$.

Mais la soft-law peut aussi représenter non pas une étape dans le processus normatif, mais le produit final ${ }^{13}$. Elle peut alors être analysée non plus comme du pré-droit, mais comme un autre

\footnotetext{
${ }^{6} \mathrm{~V}$. par ex. le rôle de l'ancienne Commission du Développement Durable dans le suivi d'Action 21.

V. D. Shelton (ed.), Commitment and Compliance. The Role of Non-binding Norms in the International Legal System, OUP, Oxford, 2000, $560 \mathrm{p}$.

${ }^{8} \mathrm{P}$. Weil, « Vers une normativité relative en droit international ? ", in RGDIP, 1982, p. 5.

${ }^{9} \mathrm{G}$. Abi Saab, «Eloge du 'droit assourdi'. Quelques réflexions sur le rôle de la soft-law en droit international contemporain », in Nouveaux itinéraires en droit, Hommage à F. Rigaux, Bruylant, Bruxelles, 1993, p. 60.

${ }_{10} \mathrm{U}$. Beyerlin, "Different types of norms in international environmental law. Policies, principles, and rules ", in The Oxford Handbook of International Environmental Law, OUP, Oxford, 2007, p. 428.

${ }^{11}$ Déclaration de Stockholm du 16 juin 1972 (Déclaration de la Conférence des Nations Unies sur l'environnement), Charte mondiale de la nature (AG-ONU, Résolution A/RES/37/7 28 octobre 1982), Déclaration de Rio du 14 juin 1992 (Déclaration sur l'environnement et le développement)

${ }_{12}$ G. Abi-Saab, "Cours général de droit international public », RCADI, t. 207, 1987, p. 210.
${ }^{13} \mathrm{G}$. Abi-Saab, « Eloge du 'droit assourdi'. Quelques réflexions sur le rôle de la soft-law en droit international contemporain », op. cit., p. 66.
} 
droit, " une variété de droit qui remplit une fonction différente de celle du droit limite; non pas le droit du justicier ou du gendarme, mais celui, plus discret et malléable, de l'architecte social » ${ }^{14}$. On pense ici à certains documents programmatoires, du type Action 21, vaste programme d'action pour le développement durable adopté lors du Sommet de Rio en 1992, pour lequel d'ailleurs un mécanisme de contrôle de la mise en œuvre a été établi. Mais documents d'orientation, plans d'action et stratégies sont également très nombreux à être adoptés à l'intérieur d'un espace conventionnel donné. De fait, certaines actions importantes trouvent leur origine dans des résolutions non contraignantes à la base d'organisations internationales (programme Man and Biosphere de I'UNESCO, Diplôme européen du Conseil de l'Europe par exemple). Que dire des Objectifs du Millénaire, essentiels et reconnus comme tels par tous mais pourtant non obligatoires ? Le Panel d'inspection de la Banque mondiale n'a-t-il pas été établi ${ }^{15}$ comme une instance indépendante au sein de la Banque mondiale, chargé d'examiner les plaintes de personnes directement affectées - ou susceptibles d'être affectées - par les projets financés par la BIRD ou l'Agence internationale pour le développement (AID), et dont le préjudice résulte d'une méconnaissance, par l'une au l'autre de ces agences, de ses politiques et procédures opérationnelles ${ }^{16}$ ?

Ajoutons que de très importantes "décisions" (de facto sinon de jure) sont prises régulièrement par les conférences des Parties (COPs), ou même par les organes qu'elles ont créés. Ainsi, le Conseil exécutif du Mécanisme de développement propre, doté de pouvoirs opérationnels importants, qui font de lui une véritable administration internationale agissante ${ }^{17}$. Chaque conférence des Parties produit un abondant droit dérivé, qui vient interpréter, préciser, appliquer le traité originel. Bien souvent, ce dernier n'est plus que la partie émergée de l'iceberg: " the majority of the norms developp through more flexible and dynamic processes, which result in formally non-legally binding decisions ${ }^{18}$.

Ainsi, sur le plan terminologique, nous proposons de distinguer «juridique », " juridiquement obligatoire » et " juridiquement contraignant ».

" Juridique » (legal en anglais) est un " adjectif qualifiant divers substantifs pour signifier qu'ils ont un rapport au droit $»^{19}$. Le contraire est non-juridique. Une obligation peut être nonjuridique, qu'on songe à une obligation morale ou religieuse...

Mais tous les instruments juridiques ne créent pas des obligations. Pour prendre un exemple, une résolution de l'Assemblée générale de l'ONU est bien un acte juridique, mais sauf exception elle ne crée pas d'obligations. L'instrument ou la norme " juridiquement obligatoire » (legally binding en anglais) crée une obligation soit un " lien juridique par lequel un sujet de droit international est tenu envers un ou plusieurs autres, d'adopter un comportement déterminé ou de s'en abstenir ${ }^{20}$. C'est un critère formel qui s'applique : la disposition est-elle consacrée par une source du droit international (convention/traité, coutume, principe général de droit, acte unilatéral liant son auteur)? Mais un traité peut aussi contenir des obligations « molles " par leur contenu. Bien qu'obligatoires sur le plan formel, ses dispositions n'obligent alors à rien de précis en substance. Leur portée pratique est de ce fait assez mince, voire nulle.

" Juridiquement contraignant " (mandatory et/ou enforceable en anglais) désigne une disposition ou un instrument pouvant être l'objet d'un acte ou d'une menace de coercition. Une mesure peut être juridiquement obligatoire mais pas contraignante (en l'absence de moyens de coercition) et c'est bien souvent le cas en droit international. Une mesure peut être contraignante aussi sans être juridiquement obligatoire parce qu'elle s'impose à l'État (comme certaines sanctions décidées par le Comité d'application du Protocole de Kyoto). On emploie aussi contraignant comme synonyme de "qui contraint à ». En ce sens, une disposition juridiquement obligatoire peut ne pas être contraignante parce que trop vague, floue ou imprécise dans sa formulation. II faut donc aussi une velléité normative pour qu'une disposition puisse avoir une incidence concrète.

\footnotetext{
${ }^{14}$ G. Abi-Saab, « Cours général de droit international public », op. cit., p. 213.

${ }^{15}$ Résolution $n^{\circ}$. BIRD 93-10 et Résolution AID n. 93-6, adoptées le 22 septembre 1993 et amendées en 1996 et 1999.

${ }^{16}$ Paragraphe 15 des Résolutions BIRD $n^{\circ}$. 93-10 et AID $n^{\circ}$. 93-6.

17 B. Kingsbury, "Global Environmental Governance as Administration. Implications for International Law ", in The Oxford Handbook of International Environmental Law, OUP, Oxford, 2007, p. 75.

${ }_{18}$ D. Bodansky, J. Brunnée, E. Hey, "International Environmental Law: Mapping the Field » », in The Oxford Handbook of International Environmental Law, OUP, Oxford, 2007, p. 21.

${ }_{20}^{19}$ Dictionnaire de droit international public, Bruylant, Bruxelles, 2001, J. Salmon (ed.), p. 629.

${ }^{20}$ Ibid., p. 765.
} 
Un mécanisme de contrôle du suivi de l'exécution par les États peut être institué pour un instrument obligatoire (traité) ou non (résolution d'organisation internationale). "Ce dernier élément ne constitue qu'un indice supplémentaire de la détermination des États ayant adopté le texte à en poursuivre la réalisation ${ }^{21}$. II peut contribuer à rendre (au moins politiquement) contraignante une résolution qui n'est pas juridiquement obligatoire. II peut donc contribuer à ce qu'elle soit mieux appliquée, que son influence soit renforcée. Mais il ne la rend pas pour autant obligatoire.

Ainsi, pour mesurer la normativité d'une disposition ou d'un instrument, nous proposons d'utiliser cumulativement trois critères :

- un critère formel (est-ce une source formelle du droit international ?) ;

- un critère substantiel (la formulation est-elle normative ?) ;

- et un critère procédural (quelles ont été les conditions d'adoption ? Quelle est l'autorité juridique et politique de l'organe qui l'a adopté(e) ? Quelle majorité a-t-elle été atteinte lors du vote? Des États ont-ils exprimé des réserves ? Si oui, lesquels ? A-t-on institué des mécanismes de suivi et/ou contrôle ?).

Prenons l'exemple de l'article 6 de la Convention sur la diversité biologique. Il prévoit que "Chacune des Parties contractantes, en fonction des conditions et moyens qui lui sont propres : (...) b) Intègre, dans toute la mesure possible et comme il convient, la conservation et l'utilisation durable de la diversité biologique dans ses plans, programmes et politiques sectoriels ou intersectoriels pertinents... ". Nous avons là un traité en vigueur, et donc juridiquement obligatoire pour les pays l'ayant ratifié. Pourtant, la disposition en question n'est pas contraignante. La formulation est trop floue et même " atténuée » par les expressions soulignées, qui en font une disposition "à la carte ". En outre, le suivi de la Convention est très léger. II repose essentiellement sur les États. II n'y a pas de procédure de contrôle effective. Nous sommes dans le formellement hard, mais le substantiellement soft.

\subsection{La forme juridique de l'accord de Paris comme résultat de quatre paramètres}

Depuis le lancement du cycle de négociations de Bali en 2007, les Parties considèrent que la forme juridique suivra la substance de l'accord. Si une telle approche a pu porter ses fruits dans d'autres domaines, il n'en reste pas moins qu'il s'avère difficile de négocier le fond tant que des incertitudes demeurent sur la forme juridique.

Les Parties doivent conduire ici une réflexion d'ensemble prenant en compte quatre paramètres :

- la forme de l'accord « cœur » lui-même, conventionnelle ou non ;

- la portée juridique des contributions nationales (INDCs) qui peut être différente de celle de l'accord « cœur»;

- les procédures destinées à assurer la transparence des actions menées nationalement, qui peuvent également inclure un volet d'assistance à la mise en œuvre ;

- les mécanismes de contrôle et réaction au non-respect.

Ces quatre paramètres ne sont bien entendu pas sans lien les uns avec les autres. Le choix effectué sur un paramètre limite ensuite les options pour les autres paramètres. À l'inverse, les faiblesses de tel choix sur un des paramètres pourront être compensées ou limitées par tel ou tel autre choix sur un autre paramètre (comme par exemple une forme non conventionnelle par des procédures de transparences renforcées). C'est bien pourquoi il faut conduire ici une réflexion globale en gardant à l'esprit que l'accord de Paris devra être à la fois robuste juridiquement, universel et équitable, dynamique et durable.

\section{La forme de l'accord " cœur»}

La feuille de route de Durban prévoit « d'élaborer au titre de la Convention un protocole, un autre instrument juridique ou un texte convenu d'un commun accord ayant valeur juridique, applicable à toutes les Parties ${ }^{22}$. C'est une alternative à deux branches qui est posée pour l'accord "cœur ", avec un choix à faire entre un protocole ou un "autre instrument juridique ou un texte convenu d'un commun accord ayant valeur juridique ».

\subsection{L'option « protocole »}

\footnotetext{
${ }^{21}$ P.-M. Dupuy, Y. Kerbrat, Droit international public, Précis Dalloz, 12ème édition, 2014, p. 428.

${ }^{22}$ Décision 1/CP.17 précitée.
} 
L'accord de Paris pourrait donc prendre la forme d'un protocole à la Convention, dont l'adoption est régie par l'article 17 de cette dernière.

Un tel protocole serait - clairement et indiscutablement - juridiquement obligatoire pour les Parties en application de la règle Pacta sunt servanda: "Tout traité en vigueur lie les parties et doit être exécuté par elles de bonne foi », règle coutumière consacrée par la Convention de Vienne de 1969 sur le droit des traités (article 26). La forme conventionnelle revêt des avantages en termes de stabilité et solidité - les engagements figurant dans un traité ne sont pas aisément réversibles, même si la décision canadienne de retrait du Protocole de Kyoto vient nous rappeler que cette possibilité existe ${ }^{23}$. Autre avantage, en tant que protocole à la Convention, il serait dans la continuité de celle-ci, faciliterait une reprise des acquis et pourrait s'appuyer sur les institutions et procédures de la Convention "mère ». En outre, le traité est généralement soumis contrairement à la décision de la COP - à la ratification des parlements nationaux ${ }^{24}$. II doit ensuite être exécuté par les Parties non seulement dans l'ordre juridique international, mais encore dans l'ordre juridique interne. Comme l'a précisé le Conseil constitutionnel français, " il appartient aux divers organes de l'État de veiller dans le cadre de leurs compétences respectives à l'application des conventions internationales dès lors que celles-ci restent en vigueur ${ }^{25}$. Sur le plan politique, c'est aussi, au moins théoriquement, une phase importante d'appropriation nationale de l'instrument international.

Le choix d'une telle option ne va pas non plus sans risque ou inconvénient. Considéré comme plus engageant, le contenu d'un traité risque fort d'être moins ambitieux que celui - par exemple - d'une décision des Parties. Ainsi, le contenu du Protocole pourrait être peu ambitieux et ne pas permettre de contenir le réchauffement à $2^{\circ}$. II s'agit là certes d'un risque indépendant de la forme juridique, mais le fait que le consensus soit requis pour l'adoption d'un protocole l'accroît considérablement: un tel accord se fait nécessairement sur la base du plus petit commun dénominateur $^{26}$. Ensuite, chaque Partie doit impérativement - puisqu'il s'agit d'un nouveau traité exprimer son consentement à être liée (par ratification, acceptation ou approbation), selon les procédures nationales, pour que le Protocole puisse lui être opposable. II y a là une marge d'incertitude. Ce nouvel instrument pourrait être peu ratifié, accepté ou approuvé. II risquerait alors de ne pas entrer en vigueur ou bien d'aboutir à un régime à deux vitesses, entre ceux qui en seraient et ceux qui n'en seraient pas (et seraient seulement Parties à la Convention-cadre). II faudra de ce point de vue prêter une attention particulière aux clauses finales, notamment à la clause spécifiant les conditions pour l'entrée en vigueur du Protocole. Les Parties ont ici l'expérience du Protocole de Kyoto qui avait posé des conditions exigeantes, requérant la ratification de 55 Parties à la Convention, représentant au moins $55 \%$ du volume total des émissions de dioxyde de carbone en $1990^{27}$. L'objectif était d'éviter que certaines Parties se retrouvent liées par un traité faiblement ratifié et donc faiblement effectif. Cette double condition explique qu'il ait fallu plus de sept ans pour que le Protocole entre en vigueur. Seule contrainte, posée par l'article 17, les Etats non Parties à la Convention ne peuvent être Parties au Protocole.

Si un nouveau Protocole était adopté, il conviendrait de déterminer le devenir du Protocole de Kyoto. En toute logique, ce nouveau Protocole devrait le remplacer. Mais comme il risque fort d'être moins contraignant, des revendications - des pays du Sud - pour le maintien du Protocole et la négociation d'une troisième période d'engagement ne peuvent être exclues. Juridiquement, le Protocole de Kyoto ne se termine pas à la fin de la deuxième période d'engagement (au 31 décembre 2020). Cependant, si une troisième période n'est pas négociée, ce qui supposerait un nouvel amendement au Protocole, sa continuation n'aurait pas grand sens puisque l'ensemble de ses dispositions ou presque visent à mettre en œuvre les engagements chiffrés des pays développés. L'extinction du Protocole n'a pas été prévue à l'origine. Elle reste toutefois possible ultérieurement d'un commun accord entre les Parties. Le droit international est ici peu formaliste. Selon la Convention de Vienne, l'extinction peut avoir lieu « b) à tout moment, par consentement

\footnotetext{
${ }^{23}$ Les Parties au Protocole disposent d'un droit de retrait aux fins de l'article 27 du Protocole. La dénonciation du Protocole prend effet à l'expiration d'un délai d'un an ou à toute date ultérieure spécifiée dans la dénonciation.

${ }^{24}$ C'est le cas général mais l'Etat, si son droit constitutionnel le permet, peut aussi signer le traité sous réserve non de ratification mais d'acceptation ou approbation, et ne pas soumettre le traité à son parlement national. C'est ainsi que les Etats-Unis ont accepté et non ratifié la Convention internationale de Minamata sur le mercure. Voir : http://www.mercuryconvention.org/Countries/tabid/3428/Default.aspx consulté le 21 février 2015.

${ }^{25}$ DC du 20 juillet 1993, Code de la nationalité, Rec. p. 196.

${ }^{26}$ En absence d'accord sur les règles de vote, la Conférence des parties n'a jamais adopté son règlement intérieur ; elle adopte toutes ses décisions par consensus.

${ }^{27}$ Protocole, art. $25 \S 1$.
} 
de toutes les parties, après consultation des autres États contractants ${ }^{28}$. Le Protocole pourrait être abrogé d'un commun accord des Parties (par une décision COP/MOP, car selon l'article 17 de la Convention "Seules les Parties à un protocole prennent des décisions en vertu dudit protocole ») ; c'est l'abrogation expresse. Mais l'abrogation peut être aussi tacite. Le droit international le prévoit en cas de conclusion d'un traité postérieur " s'il ressort du traité postérieur, ou s'il est par ailleurs établi, que, selon l'intention des parties, la matière doit être régie par ce traité » (art. 59, §1 de la Convention de Vienne). Le traité antérieur cesse alors d'exister. En l'absence d'accord international ou par sécurité dans le cas d'une abrogation tacite, les Parties pourraient aussi le dénoncer unilatéralement. La dénonciation du Protocole prendrait effet à l'expiration d'un délai d'un an ou à toute date ultérieure spécifiée dans la dénonciation ${ }^{29}$.

\subsection{L'option " un autre instrument juridique ou un texte convenu d'un commun accord ayant valeur juridique »}

Un «autre instrument juridique » ne signifie pas grand-chose, tant les termes d'« instrument " ou de "juridique " sont vagues et génériques. De même qu'on a du mal à distinguer un "instrument juridique » d'un " texte convenu d'un commun accord ayant valeur juridique ", sachant qu'un instrument juridique n'est pas forcément un instrument juridiquement obligatoire (mais peut l'être) comme nous l'avons vu. Un « texte convenu d'un commun accord ayant valeur juridique » serait nécessairement un instrument juridique. Tandis qu'un instrument juridique représentera nécessairement un "texte convenu d'un commun accord" qui aura " valeur juridique ». La version anglaise, qui distingue " another legal instrument " de l' agreed outcome with legal force ", pourrait laisser penser que, d'une part, le résultat pourrait être un paquet (y compris un protocole et des décisions COP) et d'autre part, comme certains l'ont soutenu, que ce pourrait être un accord au niveau international débouchant sur des engagements nationaux ayant eux valeur juridique. Legal force pourrait aussi faire croire à une " force » juridique donc à un instrument juridiquement obligatoire, qui serait alors plus que simplement " juridique ». Pourtant la traduction française gomme toutes ces nuances possibles dans l'interprétation.

Quoi qu'il en soit, cette formulation nous ramène finalement à deux sous-options. Se référer à tout autre instrument juridique qui ne serait pas un protocole à la Convention peut viser une modification de la Convention de 1992 et/ou de ses annexes (ou du Protocole de Kyoto, mais c'est politiquement improbable) ou encore une décision, ou un ensemble de décisions, de la COP. Juridiquement, ces deux sous-options présentent certaines différences.

\section{a. Conditions d'adoption}

La COP n'ayant pas adopté son règlement intérieur, les conditions d'adoption d'une décision de la COP sont paradoxalement plus exigeantes que celles d'un amendement à la Convention : le consensus est requis pour toute décision de la COP. S'agissant d'un amendement à la Convention, selon la procédure définie à l'article 15 de la Convention, si les Parties ne parviennent pas au consensus, il peut être adopté par un vote à la majorité des trois quarts des Parties présentes et votantes. Cela peut permettre de passer outre l'opposition d'une ou plusieurs Parties. Pour modifier une annexe à la Convention, les conditions d'adoption sont les mêmes.

\section{b. Entrée en vigueur}

La décision de la COP présente l'avantage d'être d'effet immédiat, alors qu'un amendement à la Convention doit être ratifié par les Parties et ne sera opposable qu'à celles d'entre elles qui se seront engagées et seulement quand elles l'auront fait ${ }^{30}$. Or, la ratification peut prendre beaucoup de temps, et même ne jamais intervenir. De tels processus créent le risque de régimes à deux vitesses ; certains appliquant la convention modifiée alors que d'autres continuent à appliquer le traité initial.

II est à noter qu'une procédure facilitée pour l'entrée en vigueur existe pour les annexes à la Convention (procédure non pas d'opt in, mais d'opt out), mais elle ne pourrait guère être utilisée dans la mesure où il est spécifié que les annexes "se limitent à des listes, formules et autres documents descriptifs de caractère scientifique, technique, procédural ou administratif » ${ }^{31}$. Même

\footnotetext{
${ }^{28}$ Soit des États qui ont exprimé leur consentement à être lié sans que le traité ne soit encore en vigueur à leur égard.

${ }^{29}$ Article 27 du Protocole.

${ }_{30}$ L'amendement doit en outre être ratifié par les $3 / 4$ des Parties pour entrer en vigueur.

${ }^{31}$ Convention, article 16§3, "Toute annexe adoptée en application du paragraphe 2 entre en vigueur à l'égard de toutes les
} 
si l'on envisage une annexe de type liste des contributions nationales, celle-ci est difficilement envisageable sans une modification de la Convention elle-même.

\section{c. Portée juridique}

Distinctes par leurs procédures d'adoption, la Convention modifiée ou la décision ou ensemble de décisions de la COP se différencient aussi et fondamentalement quant à leur portée. Si le traité est clairement obligatoire, la portée des décisions de la COP est ambiguë. Elles ont indiscutablement une portée pratique et opérationnelle (ou opératoire) importante et peuvent même s'imposer aux États. Que l'on songe au "paquet» de Bonn-Marrakech qui a opérationnalisé les mécanismes de flexibilité.

La Convention prévoit de ce point de vue que la COP «prend, dans les limites de son mandat, les décisions nécessaires pour favoriser l'application effective de la Convention "32. Audelà du vocabulaire utilisé, la mesure dans laquelle ces décisions pourraient créer de nouvelles obligations, être source de droit et pas seulement annoncer de futures règles ou permettre d'infléchir l'interprétation des règles existantes, demeure discutée $e^{33}$.

Une décision de la COP peut être politiquement contraignante. Mais c'est un acte juridique ("legal ») qui n'est pas juridiquement obligatoire ("legally binding ») en lui-même. Mais la Cour internationale de Justice elle-même a considéré, à propos des résolutions de l'Assemblée générale de l'ONU que "même si elles n'ont pas de force obligatoire, [elles] peuvent parfois avoir une valeur normative ${ }^{34}$. Seule une analyse casuistique, conduite disposition par disposition, permet de déterminer sa portée. II faut notamment s'interroger sur sa substance (la formulation est-elle précise, porteuse d'obligations concrètes ?) et se demander si on a assorti cette norme de mécanismes de suivi voire de contrôle.

Même non obligatoire, la décision crée en effet une situation juridique nouvelle. D'abord, un État est tenu de l'examiner de bonne foi, dans la mesure où elle reflète l'opinion de la majorité ou de tous les États parties à un traité dont il a accepté les finalités. Ensuite, la résolution revêt une valeur permissive. Un État peut par exemple, pour respecter la décision, écarter l'application d'une norme antérieure pour autant qu'il ne porte pas atteinte aux droits acquis d'autres États.

Acte juridique, la " décision » peut également devenir source de véritables obligations en elle-même. Elle est alors analysée comme un acte unilatéral d'une (quasi)organisation internationale ${ }^{35}$ dit hétéro-normateur. C'est peu probable ici, car cela doit être expressément prévu par le traité constitutif, ou résulter d'une pratique concordante. Mais elle peut aussi faire naître des obligations du fait de son acceptation expresse par les États. Cette acceptation peut être individuelle, car l'acte unilatéral de l'État est en effet une source de droit international. En effet, si, à partir d'une décision de la COP, les États s'engagent unilatéralement, mais clairement, de manière précise et inconditionnée, ils peuvent être considérés comme « tenus » internationalement ${ }^{36}$. C'est une voie pour que des "promesses " faites dans ou en vertu d'une « simple » décision de la COP engagent juridiquement l'État, autrement dit soient legally binding. ॥ faut alors que soient démontrées l'imputabilité de l'acte à un État, agissant dans les limites de sa capacité, et une publicité suffisante de la volonté de l'État. Mais il n'est pas exigé que soit établie une quelconque acceptation de l'engagement unilatéral par les autres sujets de droit.

L'acceptation peut également être collective : parce qu'un traité y renvoie (ici le Protocole) en précisant qu'elle sera formellement obligatoire, que son respect sera contrôlé, etc.

\subsection{Pour une approche combinée}

À l'heure actuelle, les Parties à la Convention se prononcent majoritairement pour l'adoption d'un protocole, qui serait complété par des décisions de la COP. Ce choix est indiscutablement le meilleur, car il permet d'optimiser les avantages et de minimiser les

\footnotetext{
Parties à la Convention six mois après la date à laquelle le Dépositaire leur en a notifié l'adoption, exception faite des Parties qui, dans le même délai, notifient par écrit au Dépositaire qu'elles n'acceptent pas l'annexe en question ».

${ }^{32}$ Article 7, nous soulignons.

${ }^{33}$ Dans l'affaire Chasse à la baleine dans l'Antarctique (Australie c. Japon), la Cour internationale de Justice a précisé récemment, à propos des recommandations de la Convention baleinière que, "Ces recommandations, adoptées sous forme de résolutions, n'ont pas force obligatoire. Cependant, lorsqu'elles sont adoptées par consensus ou à l'unanimité, elles peuvent être pertinentes aux fins de l'interprétation de la convention ou du règlement qui lui est annexé » (arrêt du 31 mars 2014, § 46).

${ }^{34}$ Avis consultatif du 8 juillet 1996, ClJ Rec. 1996, p. 254.

${ }^{35}$ R. R. Churchill, G. Ulfstein, « Autonomous institutional arrangements in multilateral environmental agreements: a little-noticed phenomenom in international law », American Journal of International Law, vol. 94, 2000, p. 623 ss.

${ }^{36} \mathrm{CIJ}$, arrêt du 20 décembre 1974, Essais nucléaires, Australie c. France, ClJ Recueil 1974, p. 267.
} 
inconvénients des différentes options. Le cœur du régime serait constitué d'un traité, forme d'engagement la plus claire et solennelle de l'État, qui fixerait les objectifs globaux et les principes de la coopération internationale renforcée. Ce traité serait complété par des décisions COP, lesquelles garantissent une sécurité juridique moindre mais permettent de gagner en souplesse, flexibilité et rapidité ${ }^{37}$. On entre ainsi dans une construction à plusieurs niveaux, qui peut permettre de préciser progressivement le contenu du traité voire de relever graduellement le niveau d'ambition. Cette décision COP ou cet ensemble de décisions ne flotteraient pas dans les limbes, mais viendraient compléter un cadre conventionnel existant selon un schéma désormais classique et largement usité dans le domaine du climat ${ }^{38}$. L'instrument conventionnel ne pourrait, quoi qu'il en soit, être l'unique pièce du futur régime. Mais si les Parties décident d'adopter un Protocole, la question qui se pose est plutôt de savoir qu'est-ce qui relèvera du droit conventionnel et de décisions de la COP. Jusqu'où ira le Protocole? Où seront inscrits les chiffres des « contributions » des États à l'effort collectif : dans le Protocole ou hors du Protocole ? Jusqu'où le Protocole organisera-t-il un suivi voire un contrôle de la mise en œuvre par les États ?

\section{Contenu et portée des " contributions déterminées au niveau national »}

\subsection{Le contenu des contributions}

Indépendamment de leur portée, la forme et le contenu des contributions s'avèrent bien sûr d'une importance majeure. II y a là un élément fondamental de leur juridicité. Plus elles seront précises, plus leur suivi sera facilité, plus un contrôle fort pourra être réalisé sur leur respect par les États. Or, rien n'est acquis de ce point de vue, puisque les contributions sont « déterminées au niveau national ».

La décision adoptée à Varsovie était extrêmement vague et porteuse d'aucune exigence particulière de ce point de vue, laissant ouvertes toutes les possibilités : des "contributions » sur l'atténuation ou l'adaptation, des "contributions" chiffrées ou non chiffrées, des périodes d'" engagement " différentes, des "contributions " en termes relatifs ou absolus, etc. Dans l'impossibilité d'avancer davantage, la Conférence de Varsovie avait demandé à la Conférence de Lima de "préciser les renseignements que les Parties communiqueront lorsqu'elles présenteront leurs contributions ${ }^{39}$. Sous des dehors techniques, la disposition cachait un véritable enjeu.

D'une part, il s'agissait de savoir si les INDCs concerneraient aussi bien l'atténuation que l'adaptation voire seulement l'adaptation. De ce point de vue, la nécessité de renforcer l'adaptation dans le futur protocole est mentionnée dans le préambule de la décision de Lima. L'adaptation fera partie, comme l'atténuation, de l'accord de Paris, au même titre que d'autres éléments et tous ces éléments seront traités de manière équilibrée (" in a balanced manner") mais non plus "à parité " comme dans une version précédente des négociations. La décision demande également aux pays développés de fournir et mobiliser un soutien financé augmenté en faveur d'actions ambitieuses d'atténuation et adaptation (\$4). De plus, les «contributions prévues déterminées au niveau national » pourront comporter un volet adaptation, mais sur une base facultative $(\S 12)$.

D'autre part, il s'agissait de préciser la forme et le contenu des INDCs en termes de réduction des émissions carbone. Ici, la conférence de Lima confirme que chaque pays doit transmettre une INDC (\$10), même si le dispositif est assoupli pour les petits pays insulaires et pays les moins avancés (\$11). Quant aux informations à fournir, c'était un enjeu important. Plus on encadre la démarche de l'État de ce point de vue, et plus on réduit sa marge de manœuvre. En d'autres termes, on réintroduit un peu de contrainte ou coordination internationale dans un processus national. Par ailleurs, plus les États sont tenus en termes de calendrier (année de référence par exemple), champ d'application, méthodologies de comptage, plus leurs INDCs seront comparables, et éventuellement évaluables de manière agrégée à l'aune de l'objectif des $2^{\circ}$. Plusieurs options subsistaient à l'ouverture de la conférence de Lima, tournant essentiellement autour de la question de savoir s'il fallait distinguer les informations pour les pays développés et en développement, ou définir la même grille pour tous. Au final, l'annexe négociée depuis des mois a tout simplement sauté. Le $\$ 10$ de la décision de Lima précise que les contributions doivent représenter une progression par rapport aux engagements en cours pré 2020 , ce qui n'est pas très

\footnotetext{
${ }^{37}$ P.-M. Dupuy et Yann Kerbrat, Droit international public, op. cit., p. 424.

${ }^{38}$ Les Accords de Bonn-Marrakech, ensemble de décisions de la Conférence des Parties soumis pour adoption à la première Réunion des Parties du Protocole en 2005, sont venus opérationnaliser le Protocole de Kyoto.

${ }^{39}$ Voir décision 1/CP.19 précitée, §2c).
} 
précis. Et c'est le $\$ 14$ seul qui apporte les précisions sur les informations à fournir. Son contenu est laconique et très ouvert. II recense quelques éléments, mais simplement à titre indicatif (" as appropriate »). Certes, les Parties doivent aussi expliquer en quoi leur INDC est équitable et ambitieuse mais à la lumière de leurs circonstances nationales, et comment elle contribue à atteindre l'objectif de la Convention. II n'empêche: les INDCs risquent fort d'être difficilement comparables et évaluables.

En termes de calendrier, la Conférence de Varsovie avait prévu que les contributions devraient être transmises "bien avant la vingt et unième session de la Conférence des Parties (d'ici au premier trimestre 2015 pour les Parties qui sont prêtes à le faire) ॥. II n'y avait pas de date butoir, laissant penser que les contributions arriveraient en ordre dispersé et pour certaines tardivement, rendant impossible toute évaluation et concertation internationale. Une date butoir a un temps été envisagée par la suite (on a parlé d'août 2015), mais la Conférence de Lima ne s'est pas accordée non plus sur ce point. Elle ne marque donc pas de progrès par rapport à Varsovie. Dans ces conditions, il ne sera pas possible d'organiser un processus de consultations multilatérales formelles sur les INDCs, pour mesurer le niveau d'ambition de l'ensemble et au besoin amener les États à augmenter leurs engagements de réduction. II a simplement été décidé que le secrétariat tiendrait à jour un registre (comme il le fait dans le cadre de l'accord de Copenhague) et publierait au 1er novembre un rapport de synthèse de l'effet agrégé des contributions des Parties qui auront été communiquées au 1er octobre $(\S 16 \mathrm{~b})^{40}$. Sachant que la conférence de Paris se tiendra du 30 novembre au 11 décembre 2015, cela laissera très peu de temps aux Parties pour éventuellement relever le niveau d'ambition. La France est aussi invitée à organiser, comme l'a fait le Pérou l'an dernier, une réunion de haut niveau sur le renforcement de la mise en œuvre de l'action climatique (\$21). Au fil des négociations, on a également perdu le mécanisme ou processus destiné à relever le niveau d'ambition du pré 2020, qui, tout en répondant à cet enjeu crucial $^{41}$, aurait pu servir de laboratoire pour le post 2020.

Ainsi, le processus de détermination des INDCs respecte-t-il pleinement les souverainetés étatiques en étant $100 \%$ national. Le "prévues" des "contributions prévues déterminées au niveau national » pouvait pourtant laisser croire que la contribution prévue et déterminée au niveau national serait l'objet d'une évaluation et éventuellement à la suite d'un réajustement, pour devenir la contribution définitive. C'est le plus gros échec de la conférence de Lima, tout au moins du point de vue de ceux qui souhaitent un accord de Paris à la hauteur des enjeux.

\subsection{Cinq options pour l'inscription des contributions}

En imaginant que le futur accord de Paris prendra la forme d'un protocole additionnel à la Convention, puisque c'est l'hypothèse la plus consensuelle aujourd'hui, cinq options peuvent être envisagées pour la reconnaissance internationale des contributions, voire leur transformation en un véritable engagement international. Le texte présente de ce point de vue une série d'options (§77) que l'on peut ramener à cinq, présentées sur le schéma suivant, impliquant des conséquences très différentes en termes de calendrier, de portée juridique des contributions, mais également s'agissant de leur suivi, contrôle et d'une éventuelle sanction du non-respect.

\footnotetext{
${ }^{40}$ On peut penser que cela correspond à une limite pour être « bien avant » la COP 21, mais ce n'est pas précisé. Voir The Emissions Gap 


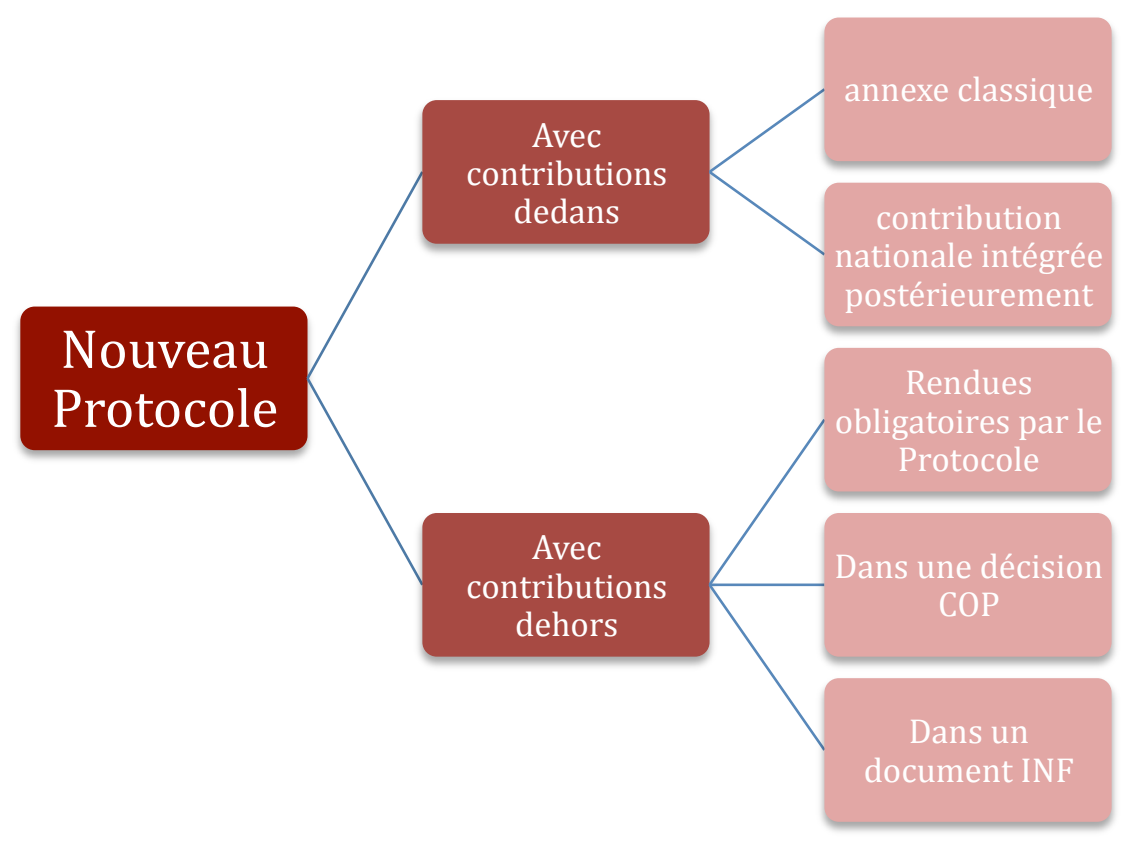

\section{a. Une annexe au protocole}

Les " contributions déterminées au niveau national " seraient alors inscrites dans une ou plusieurs annexes du protocole (options 1, 2, 3, 7 et 7 bis du texte de négociation). Ce pourrait être une seule annexe recensant l'ensemble des engagements de manière plus ou moins harmonisée, sur le modèle du Protocole de Kyoto. Cela pourrait être aussi autant d'annexes que de Parties contractantes, ce qui rendrait moins nécessaire une harmonisation même a minima, chacun pouvant présenter sa contribution comme il l'entend. Sur le plan pratique, cette approche présente l'inconvénient non négligeable d'aboutir à un texte volumineux, mais rien ne l'interdit sur un plan strictement juridique. On partirait toutefois de l'hypothèse que toutes Parties auraient l'obligation d'inscrire une contribution, aussi limitée soit-elle en termes de couverture ou d'ambition. Les contributions pourraient aussi être subdivisées en deux annexes, entre celles faites par les pays de l'Annexe I et celles des pays non Annexe I.

Dans cette hypothèse, l'annexe ou les annexes feraient partie intégrante du protocole et aurai(en)t la même nature et portée juridique, à savoir celle d'un traité juridiquement obligatoire de hard law. Leur caractère plus ou moins contraignant dépendrait de son contenu, notamment de l'existence de dispositions permettant d'assurer le contrôle du respect, voire la sanction du nonrespect.

Cette option présente plusieurs avantages. Parties intégrantes d'un traité, les " contributions " sont juridiquement obligatoires aussi bien nationalement (tout au moins pour les États parties) qu'internationalement (vis-à-vis des autres États parties). Par ailleurs, l'annexe ou les annexes serai(en)t négociée(s) et adoptée(s) en même temps que le Protocole, ce qui garantirait une forme de coordination internationale au moins minimale avant ou pendant la conférence de Paris. En effet, l'annexe ou les annexes faisant partie du protocole, elles devraient faire l'objet d'un consensus au sein de la Conférence des Parties pour être adoptées officiellement sous la forme d'une décision de cette même Conférence des Parties ${ }^{42}$. Enfin, cette option autorise en théorie des dispositions de mesure de la performance des progrès réalisés pour respecter les engagements et une procédure de contrôle en cas de non-respect, voire une procédure de règlement des différends pouvant aller jusqu'à un engagement de la responsabilité de l'Etat en cas de non-respect de ses obligations.

Cette option présente aussi deux inconvénients. D'abord, elle impose d'avoir toutes les contributions avant la Conférence de Paris pour pouvoir les formaliser en annexe(s) et les soumettre à l'adoption de la COP. II est très certainement souhaitable que les contributions

\footnotetext{
${ }^{42}$ Voir sur ce modèle la décision 1/CP.3, Adoption du Protocole de Kyoto à la Convention-cadre sur les changements climatiques (1997).
} 
nationales soient toutes sur la table à Paris, pour négocier l'accord dans un climat de confiance aussi bien vis-à-vis des contributions des autres Parties que du niveau collectif d'effort ${ }^{43}$.

Par ailleurs, une fois le traité adopté, l'approche est par définition plutôt rigide et assez peu flexible dans le temps. II serait dans cette hypothèse souhaitable de prévoir une procédure simplifiée de révision des annexes, en lien avec un éventuel mécanisme d'ambition, pour permettre à l'accord d'être évolutif au moins à la hausse. Sur le modèle du Protocole de Montréal sur l'ozone, une telle procédure dite d'ajustement a été mise en place pour modifier les engagements pris par les Parties au Protocole de Kyoto, dans le cadre de la deuxième période d'engagement. La procédure est exemplaire, car elle permet de limiter le risque de blocage (l'ajustement est considéré comme adopté par la Conférence des Parties agissant comme réunion des Parties au présent Protocole "à moins qu'un nombre supérieur aux trois quarts des Parties présentes et votantes ne fasse objection à son adoption »). En outre, son entrée en vigueur est automatique ; elle ne requiert pas de ratifications, processus toujours long et aléatoire ${ }^{44}$. Le projet d'accord lui-même prévoit une procédure assouplie pour la révision des contributions sous la forme d'une option ${ }^{45}$. II est également envisagé que les contributions figurent dans un registre en ligne, tout en étant partie intégrante de l'accord (options 7 et 7 bis). On peut imaginer qu'une telle option, relativement originale, qui revient à dématérialiser l'annexe au protocole, aurait précisément pour objet de bénéficier d'une procédure allégée de révision (par exemple ajustement automatique de la contribution d'une Partie dès lors que celle-ci le demande, tout au moins s'il s'agit de relever le niveau d'ambition de sa contribution).

\section{b. Des contributions nationales hors du Protocole, mais rendues obligatoires par le Protocole}

Comme le prévoient les options 6 et 6 bis, on peut également imaginer que les contributions nationales figurent hors du Protocole (par exemple dans un registre tenu en ligne ou non par le secrétariat sur le modèle du document INF dans lequel le secrétariat recense les " promesses » faites dans le sillage de l'Accord de Copenhague), mais que le Protocole contienne une disposition explicite rendant la soumission et le respect de ces contributions obligatoires. L'obligation pourrait être une obligation de résultat (atteindre l'objectif fixé) ou, plus raisonnablement, de moyens (mettre en œuvre tous les moyens pour s'efforcer d'atteindre cet objectif). C'est tout le sens de la récente proposition de I'AILAC ${ }^{46}$, qui mérite d'être sérieusement considérée par les Parties, car elle permet d'atteindre un équilibre intéressant entre la souplesse de contributions nationalement déterminées (qui peuvent figurer dans un simple courrier) et la sécurité juridique, chaque Partie au Protocole se trouvant internationalement liée par une obligation de mettre en œuvre le contenu de sa contribution. Autre avantage, le calendrier est assoupli, les contributions pouvant être soumises même après la conférence de Paris. Cela pourrait laisser le temps de dérouler un processus de consultation internationale, voire d'évaluation des contributions au regard de l'objectif des $2^{\circ}$. Les contributions pourraient être exigées au moment de la ratification, ou postérieurement, mais il serait alors prudent que le Protocole prévoie une date butoir.

II conviendrait aussi, dans cette hypothèse, de réfléchir aux modalités d'évolution des contributions. Pourraient-elles être revues librement par les États, à la hausse comme à la baisse ? L'option n'est alors plus guère contraignante pour les États qui, lorsqu'ils ne parviennent pas à mettre en œuvre leur contribution, peuvent la modifier à la baisse. Ou bien peuvent-ils la revoir seulement à la hausse (" non backsliding ») ? Et si oui à tout moment ou seulement lors des processus d'évaluation internationale? De la réponse à ces questions dépendrait la solidité de cette option.

\footnotetext{
${ }^{43}$ U.S. Submission, September 2014, p. 1. Australia, Submission to the Ad-Hoc Working Group on the Durban Platform for Enhanced Action (ADP) on the 2015 Agreement,| October 2014, p. 3 : " The text of the 2015 Agreement must be agreed in tandem with iNDCs and will ultimately set out the final form of post-2020 commitments ».

${ }^{44}$ Voir l'article 3 §1 quater du Protocole de Kyoto tel qu'amendé à Doha en 2012 : « (...) L'ajustement adopté est communiqué par le secrétariat au Dépositaire, qui le transmet à toutes les Parties, et il entre en vigueur le 1er janvier de l'année suivant la communication par le Dépositaire. De tels ajustements lient les Parties ».

${ }^{45}$ Voir $\$ 97.5$ (option 4).

${ }^{46}$ Independent Association of Latin America and the Caribbean AILAC Ad-Hoc Working Group on the Durban Platform for Enhanced Action (ADP), Submission on the legal architecture and structure of the elements of the 2015 Agreement, http://www4.unfccc.int/submissions/Lists/OSPSubmissionUpload/106 99 130574193142313924-

140918\%20AILAC\%20Submission\%20ADP\%202-6\%20Legal\%20Architecture,\%20vf.pdf , consulté le 22 février 2015.
} 


\section{c. L'inscription dans une décision COP}

Ce sont les options 4 et 4 bis du texte de négociation. Le protocole pourrait prévoir que les contributions des Parties seraient adoptées par une décision de la COP/MOP du nouveau protocole. Pour être immédiatement opérationnelle, cette décision pourrait être préparée par la Conférence des Parties à la Convention et soumise pour adoption à la première COP-MOP (sur le modèle des accords de Bonn-Marrakech, adoptés par la COP en 2001 et repris mutatis mutandis par un paquet de décisions de la première COP-MOP du Protocole de Kyoto). Cette option présente l'avantage de laisser un peu de temps supplémentaire aux États, autorisant par là un processus d'évaluation ex ante de leurs contributions, avec incitation à un éventuel ajustement.

Quant à la portée juridique, nous avons vu qu'une décision de la COP (et/ou de la COP/MOP) peut être politiquement contraignante, mais c'est un acte juridique ("legal ») qui n'est pas juridiquement obligatoire ( «non legally binding ») en lui-même. Malgré tout, la décision peut produire des effets juridiques.

Entérinées par des décisions de la COP/MOP, les contributions n'auraient pas la nature juridique d'un traité, mais découleraient de l'application d'un traité soumis à ratification. Reprises dans une décision COP, elles acquièrent une existence internationale et s'inscrivent dans le champ conventionnel. Les Parties sont tenues d'examiner de bonne foi la décision, outil essentiel de mise en œuvre d'un traité dont elles ont accepté les finalités. Un juge ou un arbitre pourrait même s'appuyer sur la décision pour interpréter le protocole lui-même. Enfin, les contributions pourraient faire l'objet d'une évaluation ex ante, tandis que rien n'empêcherait d'instaurer une évaluation ex post de leur respect par les États. En revanche, un État ne pourrait être sanctionné pour non-respect ; seules une assistance ou une incitation au respect sont envisageables dans ce cas de figure.

Moins solide sur le plan juridique, cette option présente également l'inconvénient d'être faiblement évolutive. Elle n'autorise pas de modification unilatérale des contributions ; une nouvelle décision COP-MOP étant requise pour modifier une décision COP-MOP. Or, le consensus est pour cela nécessaire, sauf si les Parties parvenaient à se mettre d'accord dans le cadre de l'accord de Paris sur des règles différentes pour la prise de décision par la COP/MOP (ne serait-ce que sur ce sujet-là).

\section{d. L'inscription dans un document INF}

S'agissant de cette option (reflétée par les options 5 et 5 bis du texte de négociation), les "contributions" sont compilées dans un document INF et/ou dans un registre tenu par le Secrétariat, auquel renvoie le Protocole à titre d'information, comme le sont actuellement les «promesses » de réduction pré-2020 faites en application de l'Accord de Copenhague ${ }^{47}$. Le protocole pourrait " encourager " ou " inciter » les Parties à prendre des mesures qui contribuent effectivement à la réalisation d'un objectif collectif à long terme. Mais les contributions ne seraient pas transformées en engagements internationaux. L'absence d'offre ou de contribution n'est alors pas sanctionnable (à moins que le protocole ne le prévoie). Les contributions n'ont pas d'effet, ni de portée juridique au niveau international. Leur non-respect ne peut être sanctionné (en dehors d'un shaming), tout au plus peut-on prévoir une assistance ou incitation au respect. Les contributions sont évolutives, car elles peuvent être modifiées unilatéralement par les Parties, à tout moment, à la hausse ou à la baisse (à moins que le Protocole n'interdise un backsliding). Un processus d'évaluation ex ante peut être prévu malgré tout, avec incitation à relever le niveau d'ambition, de même que des processus d'évaluation ex post.

Sous peine d'ineffectivité, le Protocole devrait dans cette hypothèse à tout le moins prévoir un objectif collectif à long terme chiffré, et des règles renforcées en matière de MRV ainsi qu'un cadre commun de comptabilisation des émissions que les Parties devraient appliquer au niveau national. Le protocole pourrait prévoir des règles renforcées sur la mesure et la communication $(M \& R)$ des émissions et leurs trajectoires et la vérification $(V)$ du respect de ces règles au titre de la transparence, ainsi qu'un processus de consultation sur les résultats de ce MRV pour encourager les Parties à revoir elles-mêmes leurs offres ou contributions. Le protocole pourrait même prévoir un mécanisme de contrôle du respect des règles MRV pour constater leur nonrespect d'un point de vue formel, avec d'éventuelles sanctions en cas de défaillance des Parties.

\footnotetext{
${ }^{47}$ On peut penser aussi au Registre/compendium des engagements « volontaires » dans le suivi de Rio + 20 (voir plateforme www.uncsd2012.org/commit ). Option soutenue par les États-Unis dans leur soumission de septembre 2014, p. 10.
} 


\section{e. Des contributions nationales intégrées au Protocole postérieurement}

Cette option n'est pas envisagée par l'actuel texte de négociation. Elle n'est pourtant pas sans intérêt. Dans ce cas de figure, des contributions des pays seraient déterminées au niveau national et inscrites dans des listes nationales ("National Schedules ») que le protocole intégrerait postérieurement à son adoption par une clause spécifique disposant qu'elles font partie intégrante de celui-ci. Les contributions nationales pourraient être construites et notifiées au Secrétariat suivant un modèle commun de présentation et une terminologie uniforme, tout en laissant aux Parties la possibilité de s'auto-différencier selon le type d'engagement (objectif chiffré absolu ou relatif, politiques et mesures, activités de projets...etc.) et le champ d'application (couvrant l'ensemble de l'économie ou sectoriel, tous les gaz à effet de serre ou seulement certains, etc.).

Sur le modèle des engagements nationaux pris dans le cadre de l'Accord général sur le commerce des services de l'OMC, cette option se distingue de la précédente en ce sens que les contributions nationales ne sont pas intégrées au Protocole dès son adoption ${ }^{48}$. Transmises par la suite, donc après la Conférence de Paris, et s'intégrant automatiquement au Protocole, elles n'ont donc pas besoin d'être acceptées par les autres États parties. Un processus de consultation ou d'évaluation ex ante - donc avant Paris - des informations fournies à l'appui de contributions des contributions demeure toutefois possible.

Moins contrainte en termes de calendrier, l'approche présente aussi davantage de souplesse, tout en conférant la même portée juridique aux contributions que si elles étaient dans une annexe classique : celle d'un traité. Une fois notifiées et faisant partie intégrante de l'accord, les contributions seraient mises en œuvre selon les dispositions de l'accord, notamment des obligations applicables à toutes les Parties (MRV, examen, contrôle). II serait possible de prévoir que les listes seraient valables pour un éventuel cycle d'engagement (5 ou 10 ans) prévu par le Protocole, et qu'elles feraient l'objet d'un examen soit continu sur base des résultats du MRV soit avant la fin de chaque cycle pour permettre aux Parties de revoir leur liste pour le cycle suivant.

Dans cette hypothèse, il resterait à déterminer si et à quelles conditions les États pourraient modifier leurs contributions. Une complète liberté, y compris pour revoir à la baisse en termes d'ambition une contribution, est théoriquement possible. Mais cela pourrait aussi être interdit ; les contributions pourraient par exemple n'être revues qu'à la hausse selon un effet cliquet (« no backsliding »). Les listes nationales pourraient être conditionnées, notamment à un soutien financier.

\section{L'enjeu du « MRV » (Measurement, Reporting and Verification) et l'évaluation des engagements}

Avec des contributions déterminées au niveau national, il est impératif d'avoir un niveau élevé de transparence dans l'accord de Paris notamment quant aux efforts fournis par chaque pays pour respecter ses engagements. C'est à ce besoin que tente de répondre les options présentées dans la section $\mathrm{J}$ du texte de négociation, qui porte sur la transparence de l'action et du soutien financier. Gage de confiance mutuelle, la transparence doit être conçue de manière à rendre le régime international sur le climat durable, ambitieux et dynamique, en reprenant les acquis en la matière pour les améliorer et les adapter en tenant compte de l'évolution des engagements. Au cœur de la discussion on retrouve la question de la différenciation, et le maintien d'un "pare-feu " (le "firewall ") entre ceux des pays développés et ceux des pays en développement avec en ligne de mire la question fondamentale de savoir si l'Accord de Paris consacrera un seul régime unique d'exigences de MRV applicable à tous ou maintiendra une différenciation comme c'est le cas de cas l'acquis hérité des Accords de Cancun (voir options 1, 2, et 3 versus option 4 sous le $\S 64$ du texte de négociation). Ces Accords de Cancún ont en effet mis en place des processus appelés "ICA " (International Consultation and Analysis) et "IAR " (International Assessment and Review), qui ont maintenu un certain degré de différenciation à travers un "pare-feu " issu de la distinction entre engagements pour les pays développés figurant à l'Annexe I de la CCNUCC et actions appropriées au niveau national («NAMAs ») pour les pays en développement non inscrits à ladite Annexe I. À cela il convient d'ajouter les modalités spécifiques de mesure et de comptabilisation des réductions d'émissions réalisées dans le cadre du mécanisme REDD+ qui ont pu être finalisées à Varsovie (2013). Enfin, les pays développés qui ont ratifié le Protocole de Kyoto doivent respecter un régime renforcé de MRV et de

\footnotetext{
${ }^{48}$ Voir aussi l'article $10 \S 1$ de la Convention de Vienne sur la couche d'ozone de 1985.
} 
comptabilisation, conformément aux articles 5,7 et 8 dudit protocole (qui reste néanmoins encore incomplet, notamment en ce qui concerne certaines activités du secteur AFOLU ${ }^{49}$ ).

II est essentiel de reprendre dans ou d'articuler avec l'accord de Paris ces acquis en matière de MRV et de comptabilisation et, en fonction du contenu de l'accord, de les améliorer Le texte de négociation ouvre ici des options variées ${ }^{50}$.

\subsection{Le régime existant}

II convient de noter que le régime MRV a déjà évolué au fil du temps, avec l'adoption des lignes directrices pour la communication et la mise en place des processus «ICA » et "IAR » qui ont permis de grandes avancées pour le suivi, la communication et surtout la vérification si on compare avec les dispositions originelles de la Convention, notamment son article 12.

Le processus "IAR », qui permet d'examiner les Communications Nationales et les rapports biannuels, y compris les inventaires nationaux, des pays développés de l'Annexe I de la CCNUCC, a formellement commencé cette année, en 2014. Ce processus d'évaluation et d'examen au niveau international consiste en " un examen technique des informations et en une évaluation multilatérale de la mise en œuvre des objectifs chiffrés de réduction des émissions pour l'ensemble de l'économie » des pays développés.

Le processus "ICA » constitue un processus de consultation et d'analyse au niveau international sur les résultats de la mesure, notification et vérification concernant les mesures d'atténuation appropriées au niveau national des pays en développement, notamment leurs rapports biannuels, dont les premiers ont été soumis en décembre 2014 selon leurs capacités et le niveau de soutien apporté pour l'établissement desdits rapports, et leurs Communications Nationales, qui doivent être soumises toutes les quatre années comme cela a été décidé à Cancún. À noter qu'une latitude particulière a été accordée aux petits États insulaires en développement et aux pays les moins avancés (PMA). La première série de consultations et d'analyses internationales débutera dans les six mois suivant la présentation de la première série de rapports biennaux actualisés par les pays en développement, soit en juin 2015. On notera que la fréquence de la participation aux séries suivantes de consultations et d'analyses internationales par les pays en développement sera déterminée par la fréquence à laquelle les rapports biennaux actualisés seront présentés. Enfin, il a été précisé à Durban (2011) que les modalités et les lignes directrices du processus "ICA » seront révisées en fonction de l'expérience acquise au cours de la première série de consultations et d'analyses internationales, au plus tard en 2017.

Le régime MRV adopté à Cancún et précisé lors de COP ultérieures devra être adapté aux dispositions de l'accord de Paris pour notamment lui donner un caractère à la fois dynamique et durable.

In fine, il conviendrait aussi d'envisager des règles de vérification qui faciliteront l'application d'un régime d'observance de nature incitative en cas de non-respect des engagements qui pourrait être déterminé par la COP informée par le Processus consultatif multilatéral établi par l'article 13 de la $\mathrm{CCNUCC}^{51}$.

\subsection{Options pour l'accord de Paris}

\section{a. L'enjeu}

Nous partons là encore de l'hypothèse d'étude que l'accord de Paris prendra la forme juridique d'un protocole additionnel à la CCNUCC. II est particulièrement important pour la robustesse de l'accord qu'il prévoit un cadre de transparence et de responsabilisation ("transparency and accountability ») qui comprendrait (voir l'objet de la transparence telle que décrite dans le $\$ 64$ du texte de négociation):

- le suivi, la communication et la vérification ( $M R V »)$ des engagements et mesures de mise en œuvre soutenus par une coopération renforcée (en matière d'atténuation, d'adaptation, de financements, de transferts de technologies, et de renforcement des capacités),

- un cadre commun de comptabilisation qui permettrait de tracer les unités utilisées par les Parties pour contribuer à la réalisation de leurs engagements et éviter le double comptage des

\footnotetext{
${ }^{49}$ Pour Agriculture, Forestry and Other Land Use.

${ }^{50}$ Voir les options 1,2 et 3 du $\S 65$ et le $\S 66$

${ }^{51}$ Voir supra.
} 
émissions, et de compter le secteur AFOLU lorsqu'il est couvert par l'engagement (voir §69 du texte de négociation).

Cet ensemble de règles pourrait être formulé dans l'accord de Paris comme des principes juridiques généraux dont les modalités et procédures de mise en œuvre seraient ensuite précisées par les Parties entre 2016 et 2020. Dans cet ensemble de règles, le cadre de transparence serait largement constitué des modalités en matière de MRV et de comptabilisation des émissions, qui auraient pour objet de suivre les progrès réalisés par les Parties pour respecter les engagements qu'elles auront déterminés au niveau national, mais également pour permettre de comparer le niveau d'efforts proposé par chacune d'elles et d'évaluer leurs contributions prises toutes ensemble (niveau agrégé d'efforts) au regard de l'ambition environnementale fixée en considération des rapports du GIEC (contenir l'augmentation des températures à $+2^{\circ}$ ).

Les modalités MRV et d'évaluation des efforts au rythme d'un cycle régulier d'engagement joueront un rôle central dans le caractère dynamique du futur accord.

\section{b. Le lien entre le MRV et l'évaluation des efforts/progrès et cycle d'engagement}

Si l'on met de côté le Protocole de Kyoto, il n'existe pas de cycle d'engagements prévu par la CCNUCC, ni d'évaluation des progrès réalisés pour respecter les engagements, pas même dans son article $4 \S 2$, b) qui parle d'abord de fourniture d'informations à intervalles périodiques sur les politiques et mesures permettant de ramener les émissions des pays développés à leurs niveaux de 1990 (à lire en parallèle avec son article 7 \$1 qui permet à la COP d'évaluer, sur la base de ces informations, "les progrès réalisés vers l'objectif ultime de la Convention ").

Mais dès lors que les engagements seront déterminés par les Parties, il convient de prévoir dans l'accord de Paris un cycle d'engagements et un processus qui serait appliqué soit en continu soit à une fréquence calée sur le rythme du cycle, pour évaluer les progrès réalisés pour respecter les engagements et les comparer entre eux, et les revoir au fil du temps, pour inciter les Parties à augmenter leur niveau d'ambition.

Différentes options sont envisageables pour le cycle d'engagements. Les propositions faites dans le texte de négociation (section K) varient entre des cycles de 5 ou 10 ans (avec éventuellement un examen à 5 ans si c'était 10 ans ou, inversement, un engagement " indicatif » à 10 ans pour donner une trajectoire à un engagement quinquennal). Les propositions varient aussi selon qu'elles concernent des engagements juridiquement obligatoires ou non. Les processus d'évaluation eux-mêmes peuvent être ou non obligatoires. La durée du cycle peut être indépendante de la fréquence de l'évaluation si celle-ci est menée en continu ou si une évaluation tous les 5 ans venait informer à mi-parcours les progrès réalisés au cours d'un cycle d'engagements d'une durée de 10 ans. En revanche, s'il est possible d'avoir une durée d'engagement différenciée selon les pays (5 ou 10 ans), il serait préférable d'avoir un processus d'évaluation qui soit le même pour tous (tous les 5 ans). En toute hypothèse, un tel cycle devrait être applicable sur le long terme, et ne pas se limiter à 2030.

La fréquence du cycle d'engagements doit bien sûr tenir compte de la fréquence avec laquelle les règles du MRV (et de comptabilisation) permettront de révéler l'information et le temps nécessaire à leur vérification, ainsi que de la fréquence des rapports du GIEC. L'accord de Paris pourrait également prévoir que le processus d'évaluation puisse être informé par d'autres sources que celles prévues par le cadre conventionnel, par exemple le MRV des émissions des transports de soute (aviation et maritime), dont les modalités sont à l'étude au sein de l'OACl et de l'OMI et en cours de négociation au niveau de l'Union européenne.

II reste aussi à déterminer sur quoi portera l'évaluation des performances au cours de chaque cycle, à savoir sur les engagements en matière d'atténuation et/ou tous les engagements, y compris les mesures d'adaptation, les financements et les transferts de technologies.

\section{c. Sur la portée du MRV}

Le caractère obligatoire du MRV sera d'autant plus nécessaire si les engagements n'ont pas de portée juridiquement contraignante au niveau international. Le MRV amélioré et unifié dans la mesure du possible devrait constituer la base d'informations vérifiées du contrôle du respect des engagements inscrits dans ou en dehors de l'accord de Paris. Si les engagements ne sont pas juridiquement obligatoires au niveau international, la COP (sur la base d'un comité de mise en $œ u v r e$ ou des conclusions du Processus de Consultation Multilatéral) ne pourrait que constater les résultats du MRV au regard de l'engagement annoncé par le pays et l'inciter à le respecter (le cas 
échéant en le privant de soutien qui lui a été accordé à cette fin, par exemple via le Fonds Vert sur le Climat, comme dans le cadre du Protocole de Montréal).

Mais restent des questions centrales qui sont de savoir quelles seront les conséquences possibles d'une telle évaluation d'un point de vue juridique (laquelle ne doit pas être confondue avec les conséquences en cas de non-respect des engagements), et quand de telles conséquences produiraient leurs effets. Ce processus d'évaluation doit d'abord être compris comme un espace d'échanges et de consultation qui doit inciter les Parties à relever leur niveau d'ambition, sur la base d'une meilleure compréhension de la nature des engagements et de l'efficacité des mesures prises par le pays pour les respecter.

II semble peu envisageable d'imposer, à la suite du processus, à un pays de relever le niveau d'ambition par une décision de la COP, laquelle ne pourrait sans doute qu'inviter ou encourager tous les pays à le faire sur la base des résultats de l'évaluation du niveau agrégé d'ambition (et en tenant compte du fossé tel que la science pourrait le révéler). Par contre, l'accord de Paris pourrait mettre en place les conditions de la comparaison des engagements en termes d'efforts, pour convaincre une Partie que d'autres font beaucoup mieux à responsabilité et capacités égales, ainsi qu'une procédure simplifiée d'ajustement de l'accord sans ratification (dans l'hypothèse où les engagements auraient une portée juridiquement obligatoire au niveau international), à l'instar de la procédure prévue par l'article 3 §1 quater du Protocole de Kyoto modifié.

Toutes ces questions nous amènent à nous interroger sur les arrangements institutionnels à prévoir pour faire de la transparence un des piliers de l'accord de Paris. Différentes options sont présentés sous le $\S 69$ du texte de négociation, qui évoque notamment "a Governing Body " à mettre en place. À l'instar du cadre pour l'Adaptation adopté à Cancun, il serait possible d'établir un cadre de travail pour la transparence dans l'accord de Paris, qui serait mis en œuvre par une nouvelle institution dédiée, le Comité de Transparence. Outre les recommandations qu'il pourrait faire à la Conférence des Parties pour améliorer et unifier progressivement les règles de MRV en fonction de l'évolution des engagements (voir options 1 et 2 du $\$ 69$ du texte de négociation), cette institution pourrait être chargée de l'évaluation technique de la performance de chaque pays (détermination des besoins/lacunes/problèmes de mise en œuvre), et de l'évaluation du niveau d'ambition de la somme des contributions/engagements au regard de l'objectif collectif à long terme (voir les liens entre sections $\mathrm{J}$ et $\mathrm{K}$ du texte de négociation).

\section{Les conséquences d'un non-respect}

Avant de réfléchir aux conséquences d'un non-respect de l'accord de Paris, il importe de rappeler ce qu'il en était de l'existant.

\subsection{Les conséquences d'un non-respect de la Convention}

"En cas de différend entre deux ou plus de deux Parties au sujet de l'interprétation ou de l'application de la Convention » s'applique la clause de règlement des différends de l'article 14 de la Convention :

- «les Parties concernées s'efforcent de le régler par voie de négociation ou par tout autre moyen pacifique de leur choix ");

- acceptation facultative (soumise à déclaration de la Partie et à réciprocité) de la juridiction de la Cour internationale de Justice (CIJ);

- ou d'une procédure d'arbitrage aboutissant à une sentence obligatoire (soumise à déclaration de la Partie et à réciprocité) ;

- à défaut commission de conciliation qui «présente une recommandation, que les parties examinent de bonne foi ».

Une telle clause n'a cependant jamais été utilisée. D'abord, les États n'ont pas fait les déclarations d'acceptation : seulement les Pays-Bas pour la CIJ et l'arbitrage, et les îles Salomon pour l'arbitrage. En raison de l'obligation de réciprocité, le seul différend qui pourrait être tranché de manière obligatoire en application de l'article 14 de la Convention serait... entre les Pays-Bas et les îles Salomon, sous la forme d'un arbitrage. De telles clauses n'ont guère de succès, car les Etats répugnent à les mettre en œuvre pour des différends multilatéraux dès lors que le nonrespect d'une convention multilatérale cause non un dommage individualisé/individualisable à un État, mais porte atteinte au bien commun ou à l'intérêt général que protège la convention. C'est en outre un acte inamical, qui fait toujours craindre un effet boomerang. 
Certes, les Parties peuvent toujours décider d'un commun accord de soumettre un différend à la Cour ou un tribunal arbitral, une fois ce différend survenu et seulement pour ce différend. Cela reste toutefois exceptionnel en particulier, là encore, pour un différend « multilatéral », même si le droit international l'autorise ${ }^{52}$.

\subsection{Les conséquences d'un non-respect du Protocole}

Le Protocole de Kyoto a donné naissance à une procédure de non-respect très élaboré qui a une vocation " de faciliter, de favoriser et de garantir le respect des engagements découlant du Protocole de Kyoto ». C'est une procédure non contentieuse de contrôle du respect et de réaction à un éventuel non-respect ${ }^{53}$. Cette procédure est sans préjudice de la procédure classique de règlement des différends figurant à l'article 14 de la Convention et s'appliquant mutatis mutandis au Protocole ${ }^{54}$.

C'est dans le cadre du Protocole de Montréal sur les substances ozonicides que la première procédure de non-respect d'une convention internationale de protection de l'environnement a été « inventée ». Procédure internationale de contrôle de la mise en œuvre et de réaction au non-respect, elle a largement contribué au succès du Protocole de Montréal. Forte de son succès, elle a depuis lors été souvent copiée, de manière plus ou moins fidèle, dans le cadre de différentes conventions internationales de protection de l'environnement. Parmi celles-ci figure le Protocole de Kyoto dont la procédure de non-respect du Protocole de Kyoto, dite de l'observance, se présente même comme la plus élaborée et innovante de ces procédures, tandis que le Comité de contrôle du respect des dispositions est le plus puissant et indépendant des comités de ce type.

Le Comité de contrôle du respect des dispositions exerce ses fonctions dans le cadre d'une plénière, d'un bureau et de deux chambres, la chambre de la facilitation et la chambre de l'exécution. Les membres du Comité siègent "à titre personnel». Ils ont "une compétence avérée dans le domaine des changements climatiques et dans des domaines pertinents tels que les domaines scientifique, technique, socioéconomique ou juridique ». Les membres de la chambre de l'exécution doivent en outre avoir une "expérience dans le domaine juridique ».

Les fonctions de la plénière sont essentiellement administratives et budgétaires. Ce sont les chambres qui traitent les cas de non-respect, et c'est le bureau du Comité qui renvoie les questions de mise en œuvre à celle des chambres compétente.

La procédure peut être déclenchée par le secrétariat, sur la base de rapports de vérification fournis par des équipes d'experts, par "toute partie à l'égard d'elle-même ", ou encore par une Partie "à l'égard d'une autre partie ". Le système est complexe, lourd, extrêmement élaboré et relativement intrusif, qu'il s'agisse du récolement de l'information (technique des registres, système des rapports) ou du traitement de l'information (vérification, compilation, communication) ${ }^{55}$. La procédure suivie, tout au moins devant la Chambre de l'exécution, est incontestablement hybride. Elle va au-delà d'une procédure diplomatique classique de type conciliation ou même médiation et présente certains traits juridictionnels ${ }^{56}$. De fait, par leur sophistication, les techniques de contrôle représentent un saut qualitatif important au regard des procédures éprouvées jusqu'alors en droit international de l'environnement, même si demeurent de nombreuses faiblesses et insuffisances, le système présentant à certains égards l'image d'un colosse aux pieds d'argile ${ }^{57}$.

\footnotetext{
${ }^{52}$ Pour ce type d'obligations que l'on peut dire «interdépendantes », la qualité de partie suffit à établir l'intérêt pour agir, dans la mesure où aucune personne tiers ou partie ne peut être isolée en tant que créancier de l'obligation (C. Santulli, Droit du contentieux international, Précis Domat Droit public, Montchrestien, 2005, p. 240). Voir le projet d'articles de la Commission du droit international sur la responsabilité des États, tel qu'adopté en 2001 (art. 48), ainsi que les commentaires de la Commission du droit international (Commission du droit international, Projet d'articles sur la responsabilité de l'État pour fait internationalement illicite et commentaires y relatifs, 2001, p. 345). Voir également l'avis du 1er février 2011 de la Chambre du Tribunal international du droit de la mer qui fait fond sur cette disposition du projet de la CDI dans laquelle elle voit un argument pour considérer que « Tout État Partie pourrait également prétendre à réparation au vu du caractère erga omnes des obligations ayant trait à la préservation de l'environnement en haute mer et dans la Zone ". TIDM, Avis consultatif du 1er février 2011, Affaire $\mathrm{N}^{\circ} 17$, Responsabilités et obligations des Etats qui patronnent des personnes et des entités dans le cadre d'activités menées dans la Zone (Demande d'avis consultatif soumise à la Chambre pour le règlement des différends relatifs aux fonds marins), § 180.

${ }_{53}$ Voir Décision 27/CMP.1 Procédures et mécanismes relatifs au respect des dispositions du Protocole de Kyoto.

${ }^{54}$ Voir son article 19.

${ }^{55}$ Cf. S. Maljean-Dubois (dir.), Changements climatiques. Les enjeux du contrôle international, La Documentation française, coll. Monde européen et international, Paris, 2007, $383 \mathrm{p}$.

${ }_{56}$ L. Boisson de Chazournes \& M. Mbengue, " À propos du caractère juridictionnel de la Procédure de non-respect du Protocole de Kyoto ", in Changements climatiques. Les enjeux du contrôle international, op. cit., p. 206.

${ }^{57}$ V. Richard, "Le système de vérification : un colosse aux pieds d'argile? ", in Changements climatiques. Les enjeux du contrôle international, op. cit., p. 337.
} 
La chambre de la facilitation (facilitative branch), pluridisciplinaire, a pour fonction de conseiller et porter assistance sur le plan technique et financier aux États rencontrant des difficultés pour remplir leurs engagements, avant même le début d'une période d'engagement. Elle doit notamment tenir compte pour ce faire du "principe des responsabilités communes, mais différenciées et des capacités respectives " des États. La démarche est avant tout pédagogique. La chambre peut faciliter l'octroi d'une aide hors de la sphère conventionnelle, mais aussi s'appuyer sur les fonds conventionnels.

La chambre de l'exécution répond à un esprit différent. La démarche est moins pédagogique et plus intrusive. Elle est en effet chargée d'établir si les Parties visées à l'annexe I respectent ou non leurs engagements chiffrés en matière de limitation et de réduction des émissions, les dispositions prévues sur le plan méthodologique et en matière de communication d'informations (inventaires, communications) et les critères d'admissibilité aux mécanismes de flexibilité. En cas de désaccord entre une Partie et les équipes d'examen composées d'experts de l'article 8, la Chambre peut aussi "appliquer des ajustements aux données d'inventaire " ou " corriger les données de compilation et de comptabilisation (...) aux fins de la comptabilisation des quantités attribuées".

Parmi les "mesures consécutives » lorsqu'un non-respect est avéré, la chambre de l'exécution peut faire une déclaration de non-respect, ce qui constitue déjà une première " sanction ». L'effet blame and shame est amplifié par la large médiatisation des Réunions des Parties. Elle peut aussi demander à la Partie concernée de présenter un plan analysant les causes et indiquant les mesures et le calendrier pour remédier à son non-respect, plan dont la mise en œuvre sera contrôlée à travers la remise régulière de rapports sur l'exécution. La chambre peut encore suspendre l'admissibilité aux mécanismes de flexibilité, si une Partie ne remplit pas les critères fixés ou si ses émissions dépassent les montants assignés. Elle ne pourra plus alors participer aux mécanismes. En outre, pour le cas où une Partie dépasse ses montants assignés, ceux de la période suivante seront réduits de 1,3 fois le montant en excès. La sanction peut donc être de taille et rares sont les conventions internationales à être allées aussi loin dans la définition d'un régime contraignant de réaction au manquement. II est à noter que ces différentes " mesures consécutives " n'ont pas un caractère punitif ; elles visent à "rétablir le respect des dispositions pour assurer l'intégrité de l'environnement et doivent inciter à ce respect ". Le terme de sanction n'est d'ailleurs jamais employé. Enfin, un recours est possible devant la COP-MOP.

Mais bien que très élaboré, le système n'est pas totalement verrouillé. D'abord, les sanctions ne sont théoriquement pas obligatoires, en l'absence d'une révision du Protocole ${ }^{58}$. Ensuite, un État en grande difficulté pourrait choisir de passer outre. Certes, il subirait l'exclusion des mécanismes de marché et serait mis "au ban " de la communauté des Parties, mais il pourrait en contrepartie cumuler les dettes environnementales de période d'engagement en période d'engagement, sachant qu'il n'y aura pas de troisième période... D'ailleurs, pas plus que les États ne sont contraints à entrer dans Kyoto, ils ne sont contraints à y rester. Ils conservent un droit de retrait que le Canada a justement fait valoir pour éviter les sanctions ${ }^{59}$. Ce retrait canadien montre combien le Comité est finalement démuni face au non-respect ${ }^{60}$.

Ajoutons que les objectifs nationaux étant largement négociés par les États, un État pouvait toujours renégocier ses objectifs de réduction de la deuxième période en intégrant le poids d'éventuelles pénalités.

Le Comité a pour l'instant traité de huit questions de mise en œuvre portant sur le nonrespect des obligations procédurales que contient le Protocole ${ }^{61}$. II examine à présent le respect par les États de leurs engagements de réduction ou limitation des émissions.

\subsection{Les conséquences d'un non-respect des accords de Cancún}

II s'agit d'un ensemble volumineux de décisions adoptées par la COP à Cancún (2010), mais aussi lors des COP ultérieures à Durban (2011) et Doha (2012) voire Varsovie (2013), dans le sillage de l'Accord de Copenhague. Leur champ est vaste puisqu'il concerne aussi bien adaptation, atténuation, financement que technologie. En eux-mêmes, les ne créent pas de

\footnotetext{
${ }^{58}$ Voir son article 18.

${ }^{59}$ Voir son article 27

${ }^{60}$ Voir Compliance Committee, CC/EB/25/2014/2 20 August 2014 Canada's withdrawal from the Kyoto Protocol and its effects on Canada's reporting obligations under the Protocol, Note by the secretariat. Montre combien le comité est démuni face au nonrespect.

${ }^{61}$ Grèce, Canada, Croatie, Bulgarie, Roumanie, Ukraine, Lituanie, Slovaquie.
} 
nouvelles obligations internationales (tout au plus viennent-ils à certains égards préciser des obligations conventionnelles). Ainsi, s'agissant de l'atténuation, en tant que telles, les «promesses » des Parties ne sont pas obligatoires en vertu du droit international ; elles figurent dans un simple document INF).

Pourtant, la mise en œuvre des Accords est "suivie »; les États doivent rendre des comptes (reporting) et une vérification a lieu. Mais un éventuel non-respect n'emporte aucune conséquence. Tout au plus un shaming si la vérification démontre certaines erreurs. Et encore n'y a-t-il aucun organe de type comité d'observance apte à ne serait-ce que constater le non-respect. La COP pourrait toutefois s'en saisir.

La difficulté est que les promesses ou réalisations sont facultatives. Une sanction risque fort d'être contre-productive en entraînant le retrait d'une promesse ou plus largement diminuant le niveau d'ambition. Dès lors, l'accent est plutôt mis sur l'incitation et le déploiement d'une assistance qui peut être juridique, technique ou financière.

\subsection{Les conséquences du non-respect du Protocole de Paris : options et enjeux}

Ces trois exemples montrent combien la forme juridique de l'accord de Paris et des contributions nationalement déterminées induisent des procédures de contrôle du non-respect différentes, avec des conséquences différentes à un éventuel non-respect.

Le texte de négociation laisse ouvertes de très nombreuses et différentes options (§88), étant précisé en note de bas de page que... "Some Parties consider that it is premature to discuss this section ". Les options vont de l'institution d'un comité ad hoc sur le modèle de celui du Protocole de Kyoto, avec deux chambres (facilitation et mise en œuvre) à la réactivation du processus consultatif multilatéral prévu à l'article 13 de la Convention ${ }^{62}$, jusqu'à l'absence de mécanisme (option 4, « No specific provisions required »).

Toujours est-il que si les contributions nationales ne sont pas dotées d'une portée juridique obligatoire, le contrôle de leur respect ne se pose plus dans les mêmes termes. Quant au nonrespect, il ne peut plus guère être sanctionné.

\section{Protocole}

a. Contributions inscrites dans le Protocole ou reconnues comme obligatoires par le

Cette option permet d'envisager une reprise de la procédure de non-respect de Kyoto qui serait vraisemblablement à atténuer notamment s'agissant de la mise en œuvre/enforcement et en particulier du volet sanction ${ }^{63}$. Une procédure plus souple et essentiellement facilitative et incitative, comme celle du Protocole de Montréal, serait sans doute plus adaptée aux vues actuelles des Parties. Des sanctions pourraient toutefois être prévues en ultime recours.

Le champ de la procédure serait à déterminer : irait-il au-delà de l'atténuation, comprenant par exemple la finance? Peut-on y inclure les mesures d'adaptation ? Beaucoup de Parties sont d'un avis contraire ${ }^{64}$. Par ailleurs, les pays du Nord et du Sud seraient-ils soumis aux mêmes obligations de ce point de vue? II faudrait aussi réfléchir à l'articulation aussi bien pratique qu'institutionnelle des travaux du Comité de non-respect avec le MRV envisagé. II faudrait enfin déterminer qui aurait le pouvoir décisionnel : la COP-MOP, le Comité, un panel ad hoc, etc. ?

\section{b. Contributions compilées dans une décision COP-MOP ou un document INF}

Dans l'hypothèse d'un protocole très bref et concis, posant seulement des principes fondamentaux, et renvoyant aux décisions COP-MOP voire à un document INF pour le reste (et notamment les contributions), il est assez difficile, voire vain, d'envisager un mécanisme fort de type observance. Pourquoi instaurer un mécanisme de contrôle hard pour contrôler du soft ? À moins d'instaurer un tel mécanisme pour contrôler le respect non des contributions nationales, mais d'autres dispositions du Protocole (les dispositions procédurales de MRV, les obligations

\footnotetext{
62 Décision 10/CP.4, Processus consultatif multilatéral (1999). Voir Sebastian Oberthur, « Options for a Compliance Mechanism in a 2015 Climate Agreement », Climate Law, 4 (2014) pp. 30-49.

${ }_{63}$ Rares sont les Parties à défendre le maintien du comité d'observance de Kyoto dans ses deux branches. Voir toutefois Submission by Nepal on behalf of the Least Developed Countries Group on the ADP Co-Chairs' Non Paper of 7 July 2014 on Parties Views and Proposal on the Elements for a Draft Negotiating Text, p. 8 ss.

${ }^{64}$ Voir par exemple Independent Association of Latin America and the Caribbean AILAC Ad-Hoc Working Group on the Durban Platform for Enhanced Action (ADP), Submission on the legal architecture and structure of the elements of the 2015 Agreement, http://www4.unfccc.int/submissions/Lists/OSPSubmissionUpload/106_99_130574193142313924-

140918\%20AILAC\%20Submission\%20ADP\%202-6\%20Legal\%20Architecture,\%20vf.pdf , précité, consulté le 28 octobre 2014.

"Committed contributions on adaptation would not be subject to the Compliance Mechanism » (§78).
} 
financières ou autres), ce qui permettrait de solidifier quelque peu un dispositif par ailleurs très fragile.

En toute hypothèse, s'agissant des contributions à l'atténuation, même si elles ne figurent que dans une décision COP-MOP, cette dernière peut passer en revue leur mise en œuvre et se saisir d'éventuelles difficultés. Elle pourrait s'appuyer sur les travaux d'un comité ou, a minima, sur un rapport du secrétariat fondé sur la vérification. Là encore se pose la question de la différenciation Nord-Sud, ou celle du couplage avec le MRV. Mais aucune sanction ne semble possible, et même un éventuel shaming par le biais d'une constatation du non-respect de sa décision par la COP-MOP semble délicat. Une assistance aux États en difficulté reste possible. Cela nécessiterait également une procédure et une institution de type comité de non-respect - qui serait peut-être dans cette hypothèse à nommer différemment, par exemple comité d'application.

S'il s'agit d'un document INF, la problématique est la même. Mais la COP-MOP est encore moins légitime à s'ingérer dans leur mise en œuvre, et tenter un shaming, risquant d'ailleurs tout simplement un retrait ou une révision à la baisse des contributions nationales. On se retrouve dans la configuration des accords de Cancún.

La question de la forme juridique doit faire l'objet d'une approche globale, prenant en compte l'ensemble des paramètres identifiés ici: la forme de l'accord "cœur " lui-même, l'inscription des contributions nationales, les mécanismes de transparence et suivi, les procédures de contrôle et de sanction du non-respect. Un consensus semble émerger en faveur d'une structure juridique hybride, avec certains éléments dans un accord central, qui pourrait prendre la forme d'un traité, et d'autres éléments qui viendraient préciser et mettre en œuvre cet accord central au-dehors, par exemple dans un ensemble de décisions qui pourraient être préparées pour adoption par la première réunion de la conférence des Parties à l'accord. Une telle structure, courante en droit international de l'environnement, et déjà expérimentée dans le cadre du régime du climat, présente d'indéniables avantages en ce qu'elle permet de trouver un équilibre entre les exigences de robustesse et durabilité, d'une part, et de flexibilité et dynamisme, d'autre part. La question de la portée des contributions nationales n'est pas tranchée non plus. L'approche ascendante de "contributions nationalement déterminées " est novatrice; elle n'est pas sans conséquence sur le plan juridique. Au regard de la faiblesse de l'encadrement international, les contributions risquent fort d'être diverses et difficilement comparables. Si un traité est effectivement adopté, seront-elles dans le traité, ou hors du traité ? Si elles sont hors du traité, ce dernier sera-t-il en mesure de leur conférer ou non une portée juridique obligatoire ? À quelles conditions les Parties pourront-elles revoir leurs contributions, à la hausse ou à la baisse ? En dépit des signaux contraires envoyés par les conférences de Varsovie et Lima, l'accord de Paris pourra-t-il faire entrer ces contributions dans des cycles de 5 ou 10 ans, ce qui présente un intérêt pratique majeur pour leur suivi et évaluation?

Outre ces différentes questions à trancher, les négociateurs auront à mettre en place un mécanisme de transparence, de suivi voire d'assistance aux Parties en éventuelle difficulté. Même si un mécanisme aussi élaboré, intrusif et contraignant que celui dit de l'observance, mis en place dans la cadre du Protocole de Kyoto, n'est plus forcément à l'ordre du jour, un mécanisme de transparence et suivi robuste sera fondamental pour assurer l'effectivité et, au-delà, la crédibilité de l'accord de Paris. C'est par là un élément destiné à renforcer la confiance des Parties. Un tel mécanisme pourrait jouer également un rôle majeur dans le renforcement progressif du niveau d'ambition. 